\title{
Resistance to cytotoxic chemotherapy-induced apoptosis in side population cells of human oral squamous cell carcinoma cell line Ho-1-N-1
}

\author{
TETSUO YAJIMA ${ }^{1,3}$, HIROMI OCHIAI ${ }^{2}$, TAKESHI UCHIYAMA ${ }^{1}$, NOBUO TAKANO ${ }^{1}$, \\ TAKAHIKO SHIBAHARA $^{1}$ and TOSHIFUMI AZUMA ${ }^{2,3}$ \\ ${ }^{1}$ Department of Oral and Maxillo-Facial Surgery, ${ }^{2}$ Department of Biochemistry, and ${ }^{3}$ HRC Project-7, \\ Tokyo Dental College, 1-2-2 Masago, Mihama-ku, Chiba 261-8502, Japan
}

Received March 13, 2009; Accepted May 19, 2009

DOI: 10.3892/ijo_00000337

\begin{abstract}
Side population (SP) cells are isolated from various tissues and cell lines based on the exclusion of DNA-binding dye Hoechst 33342 and exhibit potent stem cell characteristics. There have been few previous reports of SP cells in head and neck cancer cell lines. Thus, we isolated SP cells from oral squamous cell carcinoma cell line, Ho-1-N-1. Ho-1-N-1 contained $3.0 \%$ SP cells. Ho-1-N-1 SP cells showed selfrenewal capacity, generating both $\mathrm{SP}$ and non-SP cells. Next, we analyzed differentially expressed genes between Ho-1-N-1 SP and non-SP cells using GeneChip microarray and quantitative real-time RT-PCR. SP cells expressed high levels of ATP-binding cassette transporters with related multidrug resistance (MDR) genes. The expression of ABCB1 and ABCG2 were significantly up-regulated in Ho-1-N-1 SP cells. In addition, the expression of CFLAR, BCL2 and $B C L 2 A 1$ which are associated with anti-apoptosis, were also significantly increased in the SP cells. Chemoresistance to anticancer agents, including 5-fluorouracil and carboplatin, were compared between Ho1-N-1 SP and non-SP cells using flow cytometry and tetrazolium salt microtiter plate assay. Ho-1-N-1 SP cells survived significantly longer and SP ratio remarkably increased after anticancer agent treatment compared to non-SP cells. Immunocytochemical staining and apoptosis assay validated these results, and suggested an antiaopoptotic potential for Ho-1-N-1 SP cells. Ho-1-N-1 SP cells survived with various agents which were not only probably due to high level expression of $\mathrm{ABC}$ transporters, but also anti-apoptotic proteins. These observations indicated
\end{abstract}

Correspondence to: Dr Toshifumi Azuma, Department of Biochemistry, Tokyo Dental College, 1-2-2 Masago, Mihama-ku, Chiba 261-8502, Japan

E-mail: tazuma@tdc.ac.jp

Key words: oral squamous cell carcinoma, side population cell, cancer stem cell that Ho-1-N-1 SP cells were MDR phenotype and should be the main target for effective cancer therapy.

\section{Introduction}

Members of the ATP binding cassette (ABC) transporter family are expressed at high levels in stem and cancer cells but also have been linked to multidrug resistance (MDR) (1-6). Some ABC transporters are expressed by many cancer cells, and various types of ABC transporters contribute to drugs being pumped out of cells $(1,5,7-9)$. The most recognized of the $\mathrm{ABC}$ transporters, $\mathrm{ABCB} 1 / \mathrm{MDR} 1$ $\mathrm{P}$-glycoprotein, $\mathrm{ABCC}$ family/multidrug resistance-related protein (MRP), and ABCG2/Bcrp1, are responsible for MDR in those cancer cells (10-12). Interestingly, side population (SP) cells were isolated by dual-wavelength flow cytometry because of their capacity to efflux Hoechst 33342, which is attributable to the $\mathrm{ABC}$ transporter that is a molecular determinant of the SP cell phenotype in the hematopoietic stem cells (13-17).

Isolation method of SP cells relies on incubating the target cell population with the Hoechst 33342 and subsequent fluorescence-activated cell sorting (FACS) analysis of dualwavelength Hoechst 33342 fluorescence with gating on a specific SP that displays low red and low blue fluorescence. Non-SP cells do not efflux the Hoechst 33342 as efficiently as SP cells and on FACS analysis appear as a distinct population of cells adjacent to the SP population. SP cells are a rare subset of cells in various tissues that are highly enriched for stem cell activity and properties and that have been identified recently in multiple species and in most tissues, including bone marrow, muscle, lung, liver, prostate, skin, and mammary gland $(13,14,18-24)$.

Recently, several cancer cell lines have been found that contain a side population fractions (cancer SP cells) including the rat glioma line C6, human breast cancer cell line MCF-7, rat neuroblastoma line B104, and the human adenocarcinoma cell line HeLa (25). These repopulate both SP and non-SP cell types, indicating their multipotent differentiation potential (9). They also expressed seneral ABC transpoter proteins, providing them with increased MDR $(7,9)$. These observations indicated that cancer SP cell had cancer stem cell-like 
characteristics including MDR and tumorigenic potential. Therefore, cancer SP cells might be a candidate target for cancer treatment.

In this study, we identified cancer SP cells in a human oral squamous cell carcinoma (OSCC) cell line, Ho-1-N-1. Moreover, we evaluated the capacity for self-renewal, resistance to chemotherapeutic agents in the Ho-1-N-1 SP cells.

\section{Materials and methods}

Cell culture. The human OSCC-derived cell line Ho-1-N-1 was obtained from the Japanese Collection of Research Bioresources Cell Bank (Human Science Research Resources Bank, Osaka, Japan). Ho-1-N-1 was cultured in Dulbecco's modified Eagle's medium F-12 HAM (Sigma, St. Louis, MO, USA). The medium was supplemented with $10 \%$ fetal bovine serum (FBS) (Japan Bioserum, Tokyo, Japan) containing $100 \mathrm{U} / \mathrm{ml}$ penicillin/100 $\mu \mathrm{g} / \mathrm{ml}$ streptomycin (Invitrogen, Carlsbad, CA, USA). The cells were cultured at $37^{\circ} \mathrm{C}$ in a humidified atmosphere containing $5 \% \mathrm{CO}_{2}$ on $100 \times 20-\mathrm{mm}$ culture dishes (Corning, NY, USA).

Flow cytometry. To identify and isolate SP and non-SP fractions, Ho-1-N-1 cells were removed from the culture dish with $0.25 \%$ trypsin-EDTA (Invitrogen), pelleted by centrifugation, washed with phosphate-buffered saline (PBS), and resuspended in $37^{\circ} \mathrm{C}$ Hank's balanced salt solution (HBSS, Sigma) containing 2\% FBS and $1 \mathrm{mM}$ HEPES. The cells $\left(1 \times 10^{6}\right.$ cells $\left./ \mathrm{ml}\right)$ were stained with Hoechst 33342 at a concentration of $9 \mu \mathrm{g} / \mathrm{ml}$. The stained cells were incubated for $90 \mathrm{~min}$ at $37^{\circ} \mathrm{C}$, either alone or with $50 \mu \mathrm{M}$ verapamil for Ho-1-N-1 and then suspended in HBSS containing 2\% FBS and $1 \mathrm{mM}$ HEPES, passed through a $40-\mu \mathrm{m}$ mesh filter, and maintained at $4^{\circ} \mathrm{C}$ until flow cytometry analysis. Dead cells were excluded from flow cytometry analysis on the basis of propidium iodide staining $(2 \mu \mathrm{g} / \mathrm{ml})$ and forward scatter. Flow cytometric analysis and cell sorting were performed on a dual-laser FACS Vantage and FACS Aria (Becton-Dickinson, San Jose, CA, USA). The Hoechst 33342 was excited at $350 \mathrm{~nm}$ and its fluorescence was measured at two wavelengths using a $450 / 50 \mathrm{~nm}$ band pass filter (Hoechst blue) and a 530/30-nm band pass filter (Hoechst red). A 505-nm long pass dichroic mirror was used to separate the emission wavelengths. The gating on forward and side scatter was not stringent, and only debris was excluded.

Repopulation of SP and non-SP fraction by Ho-1-N-1 cells. To compare the self-renewal abilities of SP and non-SP cells, we cultured both cell types in supplemented medium as described previously for a day and then again sorted them into SP and non-SP cells by flow cytometry.

GeneChip microarray analysis. Total RNA was isolated from sorted Ho-1-N-1 SP and non-SP cells using Isogen (Nippon Gene, Japan). All samples were then digested with DNaseI to eliminate residual genomic DNA, and extracted with phenol:chloroform. Total RNA was then subjected liner amplification using T7-based in vitro transcription (IVT). Total RNA was reverse transcribed with an oligo-dT primer containing a T7 promoter sequence at the 5'-end (oligo-dT-T7 primer). To prime second-strand synthesis, RNA-cDNA hybrids were digested with $\mathrm{RNaseH}$, producing patches of single-strand cDNA. The second strand was filled in by DNA polymerase. The double-stranded cDNA served as a template for T7 RNA polymerase-driven IVT, which yielded up to 100x the starting mRNA pool. RNA probes were labeled in the IVT with biotinylated nucleotides. The RNA samples were converted to biotinylated cRNA and hybridized to the human Genome U133A GeneChip array (Affymetrix, Santa Clara, CA, USA). Chips were then washed and counterstained using PE-conjugated streptavidin. The scanned raw image (.DAT) and intensity (.CEL) files were analyzed using Array Assist software (Stratagene, La Jolla, CA, USA).

mRNA expression analysis. The expression levels of $A B C B 1$, $C F L A R, B C L 2$ and BCL2A1 mRNA which were recognized to up-regulate significantly by GeneChip microarray were performed with real-time quantitative reverse transcriptase polymerase chain reaction (RT-PCR). In addition, the expression of other nine $\mathrm{ABC}$ transporter genes including ABCG2, ABCC1-6, ABCC11 and ABCA2, which were associated with MDR and SP phenotype were also analyzed using TaqMan ${ }^{\circledR}$ ABI inventoried Gene Assays (Applied Biosystems, Foster City, CA, USA). The ABI inventoried TaqMan gene expression assays are listed in Table I. All self-designed gene assays followed an intron spanning design according to ABI recommendations. The RT-PCR consisted of an initial incubation at $48^{\circ} \mathrm{C}$ for $30 \mathrm{~min}$ ( $\mathrm{RT}$ reaction) followed by incubation at $95^{\circ} \mathrm{C}$ for $10 \mathrm{~min}$ and 40 cycles of $95^{\circ} \mathrm{C}$ for $15 \mathrm{sec}$ and $55^{\circ} \mathrm{C}$ for $1 \mathrm{~min}$. The transcript amount for the all genes was estimated from the respective standard curves and normalized to the amount of glycelardehyde-3phosphate dehydrogenase (GAPDH) (Pre-Developed TaqMan Assay Reagents, Applied Biosystems) transcript determined in corresponding samples. Data were collected with the Prism 7700 Sequence Detection System (Applied Biosystems).

Anticancer agent treatments. Ho-1-N-1 cells were seeded at a density of $2.5 \times 10^{4}$ cells in $100 \times 20 \mathrm{~mm}$ dishes in culture medium as described above at $37^{\circ} \mathrm{C}$ in an atmosphere containing $5 \% \mathrm{CO}_{2}$ for $24 \mathrm{~h}$. We added 5-fluorouracil (5-FU, Kyowa Hakko Kogyo, Japan), carboplatin (CBDCA, BristolMyers Squibb, NY, USA), and 5-FU+CBDCA at a concentration of $10 \mu \mathrm{g} / \mathrm{ml}$ diluted by distilled water into the culture medium and cultured for $72 \mathrm{~h}$, after which, FACS analysis was performed as previously described. The cells cultured without anticancer agent was used as a control.

We examine sensitivity to the anticancer agents of Ho-1N-1 SP and non-SP cells using a tetrazolium salt microtiter plate assay (CellTiter $96^{\circledR}$ Cell Proliferation Assay, Promega, Madison, WI, USA). SP and non-SP cells were seeded at a density of 2000 cells/well in 96-well dishes in culture medium as described previously at $37^{\circ} \mathrm{C}$ in an atmosphere containing $5 \% \mathrm{CO}_{2}$ for $24 \mathrm{~h}$ before the assay. Cells were cultured for $72 \mathrm{~h}$ in the presence of 5-FU $(10 \mu \mathrm{g} / \mathrm{ml})$, CBDCA $(10 \mu \mathrm{g} / \mathrm{ml})$, 5-FU+CBDCA combination $(10 \mu \mathrm{g} / \mathrm{ml})$ and control. At the end of the culture period, $20 \mu 1$ of 3-[4,5-dimehyl-2thiazolyl]-5-(3-carboxymethoxyphenyl)-2-(4-sulfophenyl)2H-tetrazolium inner salt (MTS) was added to each well, and 
Table I. ABI inventoried TaqMan gene expression assays and the respective $\mathrm{ABI}$ identification numbers.

\begin{tabular}{ll}
\hline Gene name & ABI ID number \\
\hline$A B C B 1$ & Hs00184491_m1 \\
$A B C G 2$ & Hs00184979_m1 \\
$A B C C 1$ & Hs00219905_m1 \\
$A B C C 2$ & Hs00166123_m1 \\
$A B C C 3$ & Hs00358656_m1 \\
$A B C C 4$ & Hs00195260_m1 \\
$A B C C 5$ & Hs00194701_m1 \\
$A B C A 2$ & Hs00242232_m1 \\
$C F L A R$ & Hs00236002_m1 \\
$B C L 2$ & Hs00153350_m1 \\
$B C L 2 A 1$ & Hs00187845_m1 \\
\hline
\end{tabular}

cells were incubated for $2 \mathrm{~h}$. The absorbance of samples was measured with a spectrophotometer at $490 \mathrm{~nm}$. The cell viability was described as a percentage over the control.

Immunofluorescence. Sorted SP and non-SP cells were grown on poly-L-lysine-coated chamber slides for $24 \mathrm{~h}$. These cells fixed with methanol for $5 \mathrm{~min}$, and permeabilized in $0.01 \%$ Triton-X100 in PBS (pH 7.4) for $10 \mathrm{~min}$. Non-specific binding was blocked with 5\% skim milk in PBS ( $\mathrm{pH}$ 7.4) for $1 \mathrm{~h}$. Primary antibodies were prepared in PBS and consisted of 1:100 rabbit anti-CFLAR (AVIVA Systems Biology, San Diego, CA, USA), 1:100 mouse anti-BCL2 (Upstate Biotechnology, Lake Placid, NY, USA) and 1:100 rabbit anti-BCL2A1 (Epitomics, Burlingame, CA, USA). Slides were incubated with primary antibodies overnight at $4^{\circ} \mathrm{C}$ and then washed three times in PBS. Secondary antibodies to the Alexa fluoro 488 (Invitrogen) consisted of 1:400. Slides were incubated with secondary antibody for $1 \mathrm{~h}$ at room temperature and washed three times in PBS. Slides were counterstained with Vectashield Mounting Media with DAPI (Vectashield ${ }^{\circledR}$, Vector Laboratories, Burlingame, CA, USA). Mounted slides were visualized using a fluorescence microscope (Axiophot 2: Carl Zeiss Co. Munchen-Hallbergmoos, Germany) at magnification x400.

Terminal deoxynucleotidyl transferase-mediated dUTP nick-end labeling assay. We evaluated the rates of apoptosis between SP and non-SP cells with four chemical reagents that induce apoptosis through different mechanisms: actinomycin D, an antineoplastic antibiotic that inhibits RNA synthesis; camptothecin, an inhibitor of nuclear topoisomerase that induces apoptosis in many types of cells; cycloheximide, an active antibiotic against many yeast and fungi that inhibits protein synthesis; and etoposide, a derivative of podophyllotoxin that inhibits topoisomerase activity. Apoptotic cells were visualized by in situ terminal deoxynucleotidyl transferase-mediated dUTP nick-end labeling (TUNEL) assay as described by the manufacturer (DeadEnd ${ }^{\mathrm{TM}}$ Fluorometric TUNEL System, Promega). The sorted Ho-1-N-1 SP and

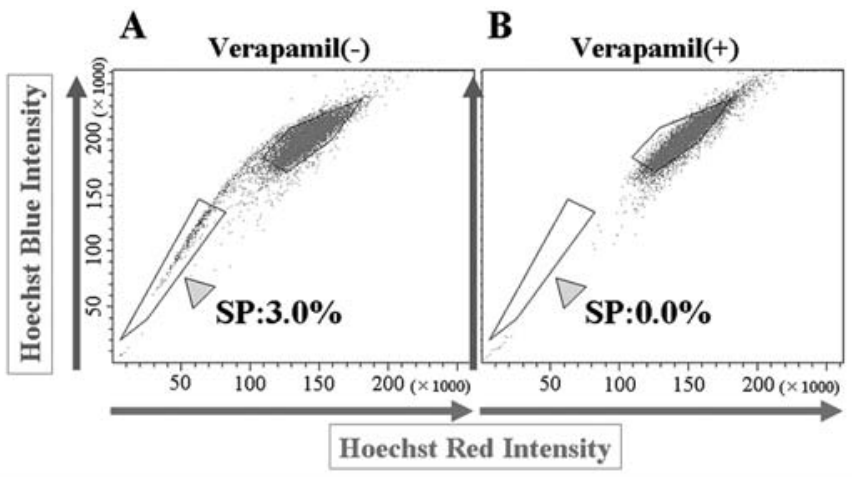

Figure 1. Typical results of the FACS analysis on Ho-1-N-1 cell line. Cells were stained with $9 \mu \mathrm{g} / \mathrm{ml}$ Hoechst 33342 and analyzed by dual-wave flow cytometry. SP cells were detected in the Ho-1-N-1 cell line. The outlined area is the SP fraction that represents $3.0 \%$ of the total cell population (A). The SP cells disappeared when cells were treated with $50 \mu \mathrm{M}$ verapamil during the staining procedure $(\mathrm{B})$.

non-SP cells were grown on coverslips for $24 \mathrm{~h}$ and treated with or without (control group) various apoptosis inducers (Ready-to-use Apoptosis Inducer Set, BioVision, Mountain View, CA, USA) including actinomycin D $(10 \mu \mathrm{M})$, camptothecin $(2 \mu \mathrm{M})$, cycloheximide $(100 \mu \mathrm{M})$, and etoposide $(100 \mu \mathrm{M})$ for $48 \mathrm{~h}$. After exposure, the cells were fixed in $4 \%$ methanol-free formaldehyde solution ( $\mathrm{pH} 7.4$ ) for $25 \mathrm{~min}$ at $4^{\circ} \mathrm{C}$. After permeabilizing the cells in $0.2 \%$ Triton X-100 solution for $5 \mathrm{~min}$, the cells were incubated with $\mathrm{rTdT}$ buffer for $60 \mathrm{~min}$ at $37^{\circ} \mathrm{C}$. The reaction was terminated using 2X SSC for $15 \mathrm{~min}$ at room temperature. The cells were washed, mounted in Vectashield (Vector Laboratories), and photographed using a Nikon fluorescent microscope. The number of apoptotic cells per high power field was counted. The mean percentage of apoptosis positive cells was determined in at least five random fields at magnification x400 in each sample.

Statistical analysis. Data are expressed as means \pm SD. Statistical analysis was performed by Mann-Whitney U test, with significance assigned at the $\mathrm{P}<0.05$ and $\mathrm{P}<0.01$ levels.

\section{Results}

FACS analysis. SP cells were isolated from Ho-1-N-1 based on the ability of the cell population to efflux Hoechst 33342. When stained cells were observed simultaneously at dual wavelengths (red and blue), typical distinct populations were identified (Fig. 1A). We observed that SP population of cells (outlined in Fig. 1A) represented $3.0 \%$ of the total cells, while verapamil-treated cells had no such population (Fig. 1B). The ability of the SP cell populations to efflux Hoechst 33342 was inhibited by the calcium channel blocker verapamil.

Repopulation of SP and non-SP fractions by Ho-1-N-1 cells. We found that the cultures initiated with SP cells contained SP and non-SP cells, whereas those with non-SP cells generated only non-SP cells (Fig. 2). The percentage of SP cells increased when analyzed after a week of culture of the SP cells compared to that of the pre-isolated Ho-1-N-1 cell line (pre-isolation vs. 1st sort and a 1 week culture: 3.0 vs. $10.3 \%$ ) (Fig. 2B). In 
Pre-isolation

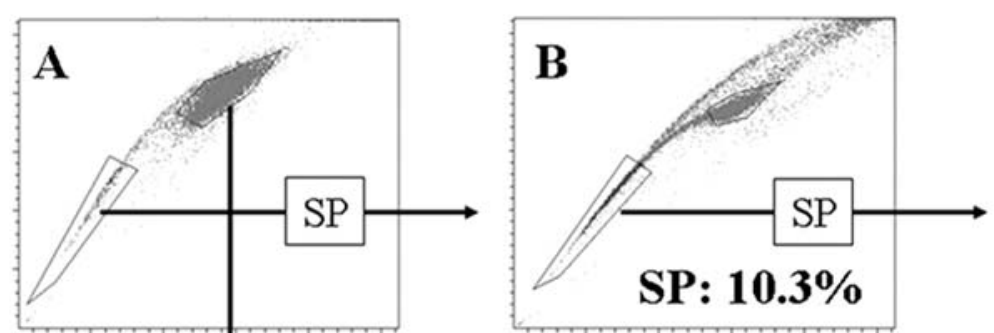

3rd sorted SP

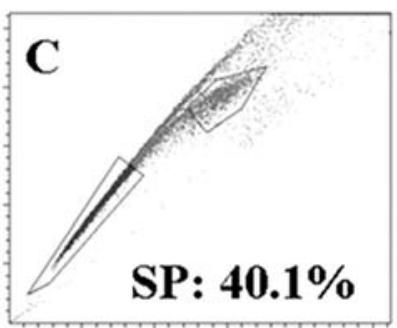

\section{1st sorted non-SP}

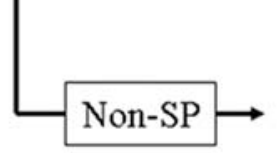

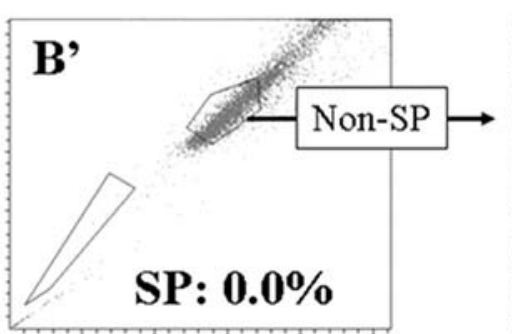

\section{3rd sorted non-SP}

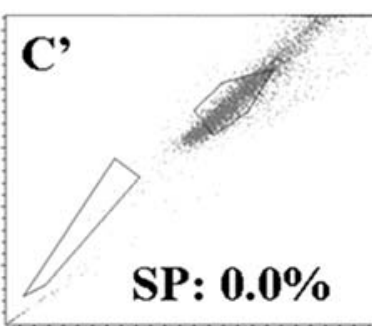

Figure 2. SP cells display a capacity for self-renewal. The Ho-1-N-1 cell line contains 3.0\% SP cells (A). After the isolation, both the Ho-1-N-1 SP and nonSP fractions were cultured for 1 week. SP and non-SP cells were reanalyzed by flow cytometry (B and B'). The SP cells maintain the SP subpopulation $(10.3 \%)$ whereas no SP cells were found in the fraction initiated from non-SP cells. When the sorting and culture were repeated three times $\left(\mathrm{C}\right.$ and $\left.\mathrm{C}^{\prime}\right)$, the percentage of SP cells increased greatly (40.1\%). However, the non-SP cells generated only non-SP cells.

Table II. Genes regulated >5-fold between SP and non-SP cells in Ho-1-N-1.

\begin{tabular}{llll}
\hline Gene symbol changes & Gene name
\end{tabular}

Transporter

$A B C B 1$

Cell death

BCL2

CFLAR

$B C L 2 A 1$
B-cell CLL/lymphoma 2

56.1

Caspase- 8 and FADD-like apoptosis regulator

BCL2-related protein A1
39.2

5

Biologic functions of the up-regulated genes determined by the GeneChip microarray. We selected the tumorigenetic-related genes into two categories except for the genes with unknown functions.

addition, when the sorting and the 1 week culture was repeated 3 times, the percentage of SP cells greatly increased compared to that of the 1 st sort and the 1 week culture (1st sort and culture vs. 3 times repeated sorting and culture: $10.3 \%$ vs. $40.1 \%$ ) (Fig. 2C). However, the non-SP cells generated only non-SP cells when the sorting and culture were repeated (Fig. 2B' and $\mathrm{C}^{\prime}$ ). We performed analyses 3 times with similar results in each case.

Gene expression analysis. Using an Affymetrix HG-U133A GeneChip array, we determined differentially expressed genes between Ho-1-N-1 SP and non-SP. We identified 148 genes which were up-regulated $>5.0$-fold in the SP cells compared to the non-SP cells. We categorized the specifically up-regulated tumorigenic genes in the SP cells according to their biologic functions. We listed four tumorigenic genes significantly up-regulated in the SP cells into two categories except for the genes with unknown functions (Table II). In particular, the expression of ABCB1 (45-fold) related to MDR was up-regulated in the SP cells. The expression of genes related to cell death, such as BCL2 (56.1-fold), CFLAR (39.2-fold) and BCL2A1 (5-fold) were also increased.

Next, we performed real-time quantitative RT-PCR analysis. Ho-1-N-1 SP cells expressed higher levels of the $\mathrm{ABC}$ transporter genes than did the non-SP isolated from the same original cells (Fig. $3 \mathrm{~A}$ ). In particular, $A B C B 1$ and 

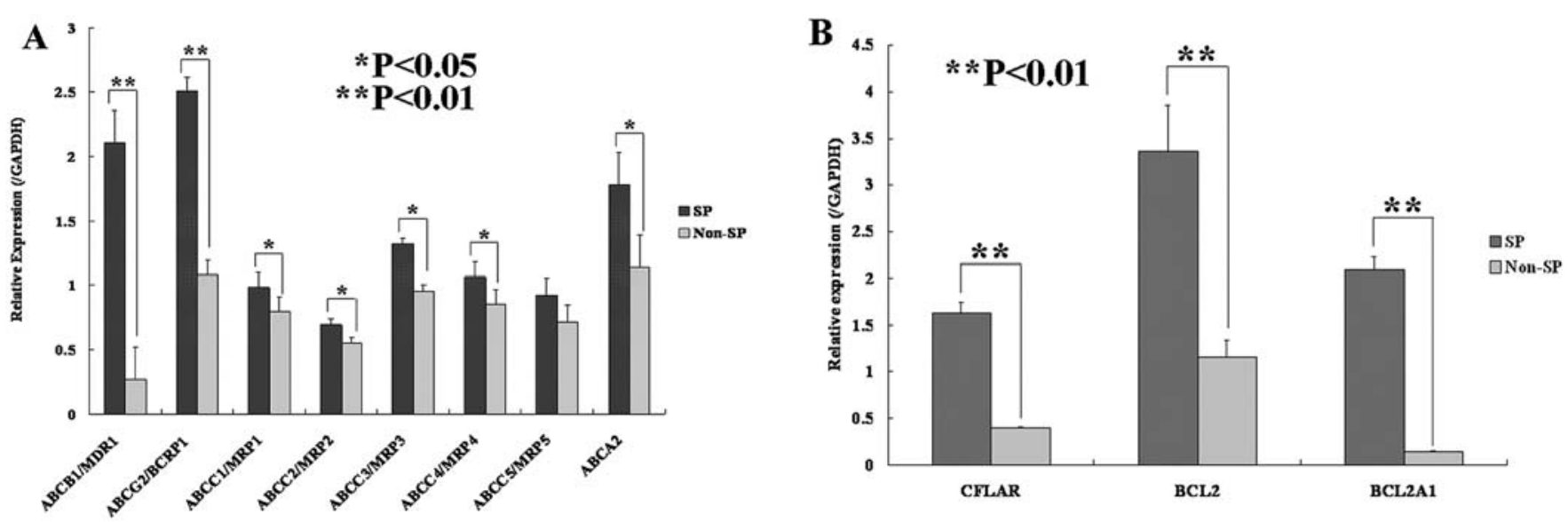

Figure 3. Quantification of mRNA levels between Ho-1-N-1 SP and non-SP cells by real-time RT-PCR. SP cells showed high expression of most ABC transporter genes than non-SP cells (A). In particular, there were significant differences in ABCB1 and ABCG2 expression between SP and non-SP cells. The SP cells also expressed high levels of CFLAR, BCL2 and BCL2A1, which was related to anti-apoptosis more than the non-SP cells (B). Data are expressed as means \pm SD.

$A B C G 2$ expressed significant differences $(\mathrm{P}<0.01)$ between SP and non-SP cells. However, ABCC6 and ABCC11, which are major transporters for 5-FU were undetectable in both Ho-1-N-1 SP and non-SP cells (data not shown). In antiapoptotic related genes, a significant difference was seen in CFLAR, BCL2 and BCL2A1 between SP and non-SP (Fig. $3 \mathrm{~B})$. These results corresponded very well to the microarray data. The data are expressed as the mean $\pm \mathrm{SD}$ of three independent experiments with samples in triplicate.

Ho-1-N-1 SP cell resistance to various anticancer agents. The presence of high levels of ABC transporter proteins indicated that SP cells might have enhanced efflux capacity not only for Hoechst 33342 but also for anticancer agents, including those used to treat OSCC. The percentage of SP cell was much higher in the anticancer agent-treated group than in the control (Fig. 4A). We also found that simultaneous treatment with two anticancer agents resulted in decreased percentage of SP cell compared to single treatment. In microtiter plate assay, after exposure to anticancer agents, the viability of the Ho-1-N-1 SP cells was significantly higher than that of the non-SP cells (Fig. 4B). Data are presented as a percentage of proliferation of treated cells compared to control cells and are the mean of three independent experiments, each performed in triplicates.

Immunofluorescent analysis. We performed immunofluorescent microscope observations to evaluate protein expression of CFLAR, BCL2 and BCL2A1. Corresponding to the observed increased gene-expression in the microarray and real-time RT PCR analysis, staining for CFLAR, BCL2 and BCL2A1 were positive in SP cells and negative in non-SP cells (Fig. 5). The CFLAR, BCL2 and BCL2A1 proteins in the Ho-1-N-1 SP cells were surrounded by obvious green fluorescence; no fluorescence was seen in non-SP cells. mRNA status was matched to the protein expression values in the immunofluorescence analysis.

Apoptosis assay. We examined four different chemical reagents. Forty-eight hours after treatment, the number of TUNEL-positive cells in Ho-1-N-1 SP cells treated with

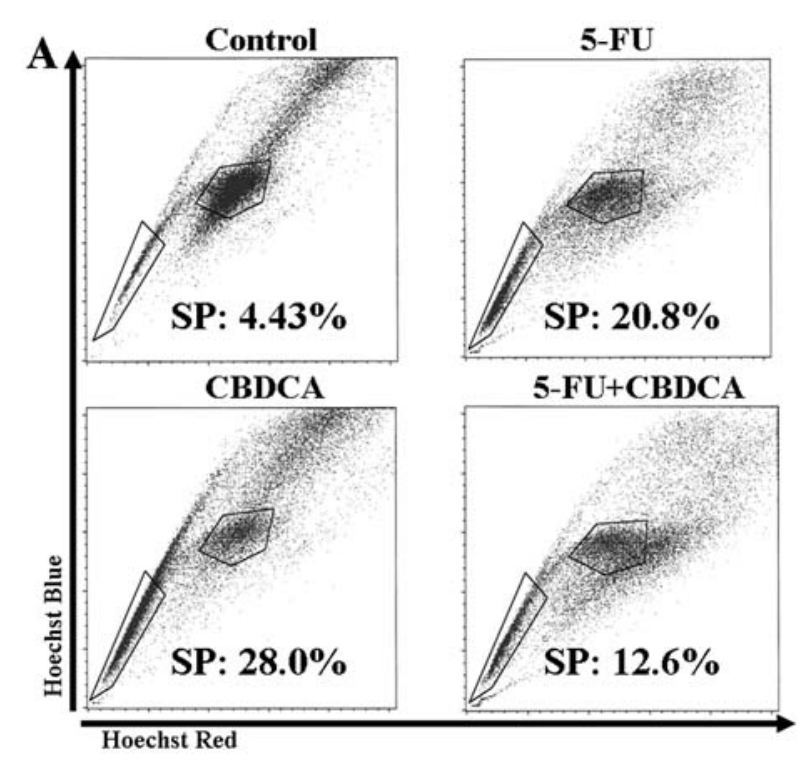

B

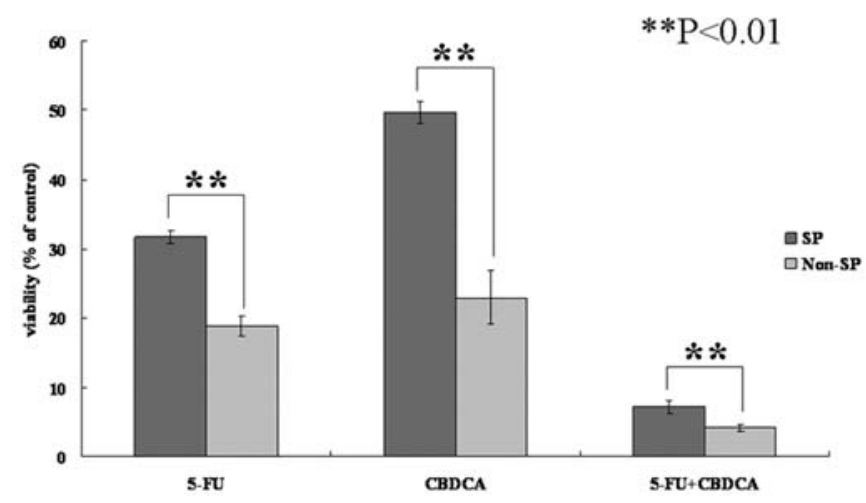

Figure 4. FACS analysis on SP cells which were treated with anticancer agents. The Ho-1-N-1 cell line was cultured in the presence of $10 \mu \mathrm{g} / \mathrm{ml} 5-\mathrm{FU}$, carboplatin, and 5-FU+carboplatin for $72 \mathrm{~h}$ and then analyzed for the SP cells (A). Regarding all cell groups exposed to anti-neocancer agents, the percentage of SP cell increased compared with the control. Combined treatment using anticancer agents decreased the percentage of SP cell compared with the group in which one treatment was used. In microtiter plate assay, after exposure to anticancer agents, the viability of the SP cells was markedly higher compared to that of the non-SP cells (B). No treatment group was used as a control group. Data are expressed as means $\pm \mathrm{SD}$. 


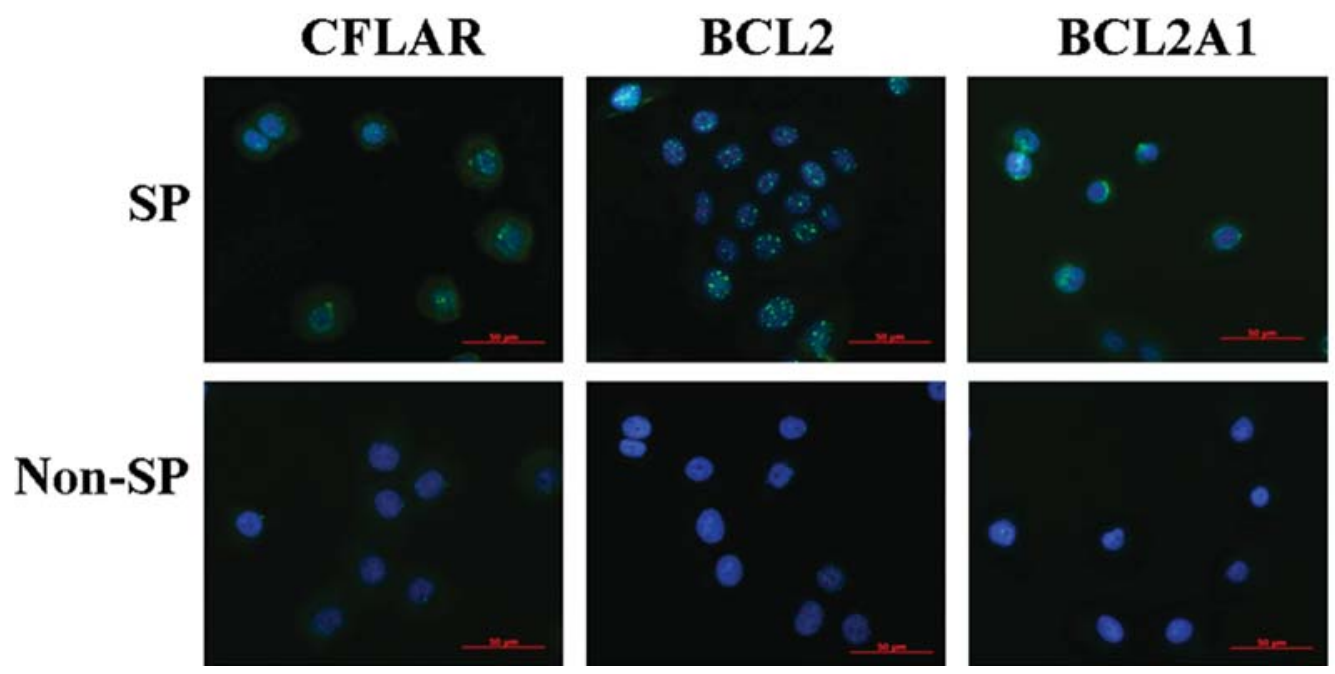

Figure 5. Immunocytochemical staining to examine the anti-apoptotic potential of Ho-1-N-1 SP cells. Both SP and non-SP cells were cultured as described for $24 \mathrm{~h}$ and then stained with anti-apoptotic marker CFLAR, BCL2 and BCL2A1. All proteins in the SP cells are surrounded by green fluorescence; fluorescence is not seen in the non-SP cells. Scale bar, $50 \mu \mathrm{m}$.

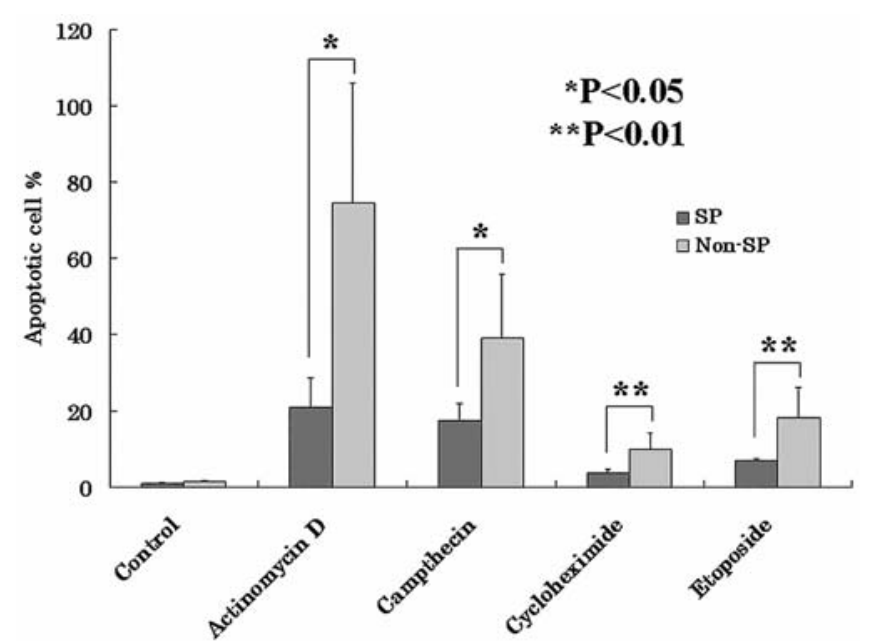

Figure 6. Apoptosis assay. The number of TUNEL-positive cells in the Ho-1-N-1 SP cells treated with or without (control) apoptosis inducers was significantly lower than in the non-SP cells. Data are expressed as means $\pm \mathrm{SD}$.

apoptosis inducers was significantly lower than with non-SP cells (Fig. 6). The SP cells were significantly resistant to each reagent.

\section{Discussion}

Head and neck cancer, including oral cancer, is the sixth most common cancer in the world. The most common type of head and neck cancer is squamous cell carcinoma (SCC), however, not many previous reports exist on SP cells in SCC (26). This is the first report describing the high frequency of SP cells in OSCC cell line Ho-1-N-1 and showed that SP cells were anti-apoptotic.

First, we identified $3.0 \%$ SP cells in Ho-1-N-1 cell. Next, we examined whether the Ho-1-N-1 SP cells reflect the biological characteristics of stem-like cells, such as self- renewal and multilineage proliferation. We found that SP cells reproduced both SP and non-SP cells, whereas non-SP cells generated only non-SP cells. These results indicate that, at least in Ho-1-N-1, a tumor hierarchy exists in which SP cells can generate both SP and non-SP cells; this agrees with previous observations that the SP fraction can divide asymmetrically and display a capacity for self-renewal $(7,9,25)$.

Gene expression studies have been used widely to stratify tumors and identify informative signatures to discover pathogenetic molecules and/or pathways (27). We analyzed differentially expressed genes between Ho-1-N-1 SP and non-SP cells using a GeneChip microarray. We identified 148 genes which were up-regulated $>5$.0-fold in the SP cells compared to the non-SP cells. Ho-1-N-1 SP cells expressed high levels of $A B C B 1$ related to MDR and anti-apoptotic genes such as $C F L A R, B C L 2$ and $B C L 2 A 1$. In quantitative real-time RT-PCR, the expression levels of several ABC transporter genes including $A B C B 1 / M D R 1$ and $A B C G 2 /$ $B c r p 1$, which are associated with MDR, were significantly up-regulated in Ho-1-N-1 SP cells. ABCB1 up-regulation has been associated with clinical resistance to anticancer agents (including anthracyclines, taxanes, epipodophyllotoxins, and vinca alkaloids) and worsened outcomes in a number of human malignancies $(1,2,28,29)$. Several previous studies indicated that $\mathrm{ABCG} 2$ is the key transporter for pumping out Hoechst $33342(8,13,14)$. The drug transport property conferred by the ABCG2 transporter is an important marker in the isolation and analysis in hematopoietic stem cells and termed 'side population' cells $(8,13,14)$. In our research we found that not only $A B C B 1$ and $A B C G 2$, but other $\mathrm{ABC}$ transporters were significantly up-regulated, which might be an explanation for the MDR phenotype.

The association of increased expression of ABC transporters in cancer SP cells with resistance to chemotherapeutic agents has been reported $(7,9)$. We examined the sensitivity of Ho-1-N-1 SP cells to 5-FU and CBDCA commonly used in the treatment of OSCC by FACS analysis and tetrazolium salt microtiter plate assay. After 72-h exposure, these agents 
depress total cell growth, but the viability of the SP cells was significantly higher than that of the non-SP cells. However, the combination of anticancer agents with a different efflux mechanism decreased the percentage of SP cells. It was suggested that some of the survived SP cells with 5-FU treatment could be killed with CBDCA. These results indicated that combination usage of several anticancer agents could decrease SP cell survival and could end up with better treatment outcome. But percentage of SP was still found much higher than control group (almost 3-fold). Furthermore, ABCC11 related to efflux of 5-FU was not detected in Ho-1N-1 cells. Nevertheless, Ho-1-N-1 SP cells showed higher resistance to 5-FU than non-SP cells. Data from this research provides insight into the existence of key molecules, which may be associated with MDR not only through ABC transporters but also through other mechanisms, possibly associated with increased expression of anti-apoptotic genes such as CFLAR, BCL2 and BCL2A1.

The expressions of CFLAR, BCL2 and BCL2A1, which are associated with anti-apoptosis, also were significantly increased in Ho-1-N-1 SP cells. CFLAR binds to Fas-associated death domain (FADD) inhibiting caspase- 8 activation. Several studies have shown that down-regulating CFLAR not only sensitizes various tumor cells to death ligand-induced apoptosis but also synergistically enhances chemotherapy-induced apoptosis (30-32). CFLAR overexpression protected colorectal cancer cells from the growth-inhibiting effects of combined 5-FU and oxiliplatin (33). BCL2 family, including BCL2A1 prevented loss of mitochondrial membrane potential, discharge at cytochrome $\mathrm{c}$ and caspase-9, but not caspase- 8 activation. $B C L 2$ family genes are known to be key regulators and major inhibitors of apoptosis. The human BCL2A1 gene encodes for a BCL2 family protein, the expression of which in normal tissue largely is restricted to the hematopoietic compartment, but is overexpressed in certain human non-hematopoietic malignancies such as skin SCC and melanoma $(34,35)$.

Moreover, we could envision a model in which Ho-1-N-1 SP cells reveal high survival ability through induction of CFLAR, BCL2 and BCL2A1 expression by immunofluorescense and TUNEL assay. These anti-apoptotic proteins were necessary for survival of Ho-1-N-1 SP cells. Our results suggest that Ho-1-N-1 SP cells not only overexpress ABC transporters but also anti-apoptotic proteins that may be more resistant to the various anticancer agents and survive better than non-SP cells.

In conclusion, we showed that it is possible to apply the isolation method of tissue stem cells, side population cells, to the OSCC cell line, Ho-1-N-1. This study confirms that Ho1-N-1 SP cells have CSC-like properties such as self-renewal and MDR including anti-apoptosis, which paves the way for the discovery of new targets for alternative therapeutic strategies. It is suggested that targeting a cancer SP cell might be a new index in use of anticancer agents. Future chemotherapeutic strategies should therefore consider eradicating this high MDR population.

\section{Acknowledgements}

We thank Ms. A. Yokoyama for her excellent technical assistance. This study was supported by Grant HRC7 from the
Oral Health Science Center of Tokyo Dental College and a 'High-Tech Research Center' Project for Private Universities.

\section{References}

1. Huang Y, Anderle P, Bussey KJ, et al: Membrane transporters and channels: role of the transportome in cancer chemosensitivity and chemoresistance. Cancer Res 64: 4294-4301, 2004.

2. Abbott BL: ABCG2 (BCRP) expression in normal and malignant hematopoietic cells. Hematol Oncol 21: 115-130, 2003.

3. Schatzlein AG: Delivering cancer stem cell therapies - a role for nanomedicines? Eur J Cancer 42: 1309-1315, 2006.

4. Guo Y, Kotova E, Chen ZS, et al: MRP8, ATP-binding cassette $\mathrm{C} 11$ (ABCC11), is a cyclic nucleotide efflux pump and a resistance factor for fluoropyrimidines 2',3'-dideoxycytidine and 9'(2'-phosphonylmethoxyethyl)adenine. J Biol Chem 278: 29509-29514, 2003.

5. Frank NY, Margaryan A, Huang Y, et al: ABCB5-mediated doxorubicin transport and chemoresistance in human malignant melanoma. Cancer Res 65: 4320-4333, 2005.

6. Larsen AK, Escargueil AE and Skladanowski A: Resistance mechanisms associated with altered intracellular distribution of anticancer agents. Pharmacol Ther 85: 217-229, 2000.

7. Haraguchi $\mathrm{N}$, Utsunomiya $\mathrm{T}$, Inoue $\mathrm{H}$, et al: Characterization of a side population of cancer cells from human gastrointestinal system. Stem Cells 24: 506-513, 2006.

8. Patrawala L, Calhoun T, Schneider-Broussard R, et al: Side population is enriched in tumorigenic, stem-like cancer cells, whereas $\mathrm{ABCG} 2^{+}$and $\mathrm{ABCG} 2^{-}$cancer cells are similarly tumorigenic. Cancer Res 65: 6207-6219, 2005.

9. Hirschmann-Jax C, Foster AE, Wulf GG, et al: A distinct 'side population' of cells with high drug efflux capacity in human tumor cells. Proc Natl Acad Sci USA 101: 14228-14233, 2004.

10. Belinsky MG, Chen ZS, Shchaveleva I, et al: Characterization of the drug resistance and transport properties of multidrug resistance protein 6 (MRP6, ABCC6). Cancer Res 62: 6172-6177, 2002.

11. Borst P and Elferink RO: Mammalian ABC transporters in health and disease. Annu Rev Biochem 71: 537-592, 2002.

12. Litman T, Brangi M, Hudson E, et al: The multidrug-resistant phenotype associated with overexpression of the new ABC half-transporter, MXR (ABCG2). J Cell Sci 113: 2011-2021, 2000.

13. Goodell MA, Brose K, Paradis G, Conner AS and Mulligan RC: Isolation and functional properties of murine hematopoietic stem cells that are replicating in vivo. J Exp Med 183: 1797-1806, 1996.

14. Goodell MA, Rosenzweig M, Kim H, et al: Dye efflux studies suggest that hematopoietic stem cells expressing low or undetectable levels of CD34 antigen exist in multiple species. Nat Med 3: 1337-1345, 1997.

15. Zhou S, Schuetz JD, Bunting KD, et al: The ABC transporter $\mathrm{Bcrp} 1 / \mathrm{ABCG} 2$ is expressed in a wide variety of stem cells and is a molecular determinant of the side-population phenotype. Nat Med 7: 1028-1034, 2001.

16. Scharenberg CW, Harkey MA and Torok-Storb B: The ABCG2 transporter is an efficient Hoechst 33342 efflux pump and is preferentially expressed by immature human hematopoietic progenitors. Blood 99: 507-512, 2002.

17. Krishnamurthy P, Ross DD, Nakanishi T, et al: The stem cell marker Bcrp/ABCG2 enhances hypoxic cell survival through interactions with heme. J Biol Chem 279: 24218-24225, 2004.

18. Rivier F, Alkan O, Flint AF, et al: Role of bone marrow cell trafficking in replenishing skeletal muscle SP and MP cell populations. J Cell Sci 117: 1979-1988, 2004.

19. Meeson AP, Hawke TJ, Graham S, et al: Cellular and molecular regulation of skeletal muscle side population cells. Stem Cells 22: 1305-1320, 2004

20. Majka SM, Jackson KA, Kienstra KA, et al: Distinct progenitor populations in skeletal muscle are bone marrow derived and exhibit different cell fates during vascular regeneration. J Clin Invest 111: 71-79, 2003.

21. Summer R, Kotton DN, Sun X, et al: Side population cells and Bcrp1 expression in lung. Am J Physiol Lung Cell Mol Physiol 285: 97-104, 2003.

22. Pearce DJ, Ridler CM, Simpson C, et al: Multiparameter analysis of murine bone marrow side population cells. Blood 103: 2541-2546, 2004. 
23. Montanaro F, Liadaki K, Volinski J, et al: Skeletal muscle engraftment potential of adult mouse skin side population cells. Proc Natl Acad Sci USA 100: 9336-9341, 2003.

24. Alvi AJ, Clayton H, Joshi C, et al: Functional and molecular characterisation of mammary side population cells. Breast Cancer Res 5: 1-8, 2003.

25. Kondo T, Setoguchi T and Taga T: Persistence of a small subpopulation of cancer stem-like cells in the C6 glioma cell line. Proc Natl Acad Sci USA 101: 781-786, 2004.

26. Hunter KD, Parkinson EK and Harrison PR: Profiling early head and neck cancer. Nat Rev Cancer 5: 127-135, 2005.

27. Venezia TA, Merchant AA, Ramos CA, et al: Molecular signatures of proliferation and quiescence in hematopoietic stem cells. PLoS Biol 2: e301, 2004.

28. Demidenko ZN, Halicka D, Kunicki J, et al: Selective killing of adriamycin-resistant (G2 checkpoint-deficient and MRP1expressing) cancer cells by docetaxel. Cancer Res 65: 4401-4407, 2005.

29. Dean M, Fojo T and Bates S: Tumour stem cells and drug resistance. Nat Rev Cancer 5: 275-284, 2005.

30. Fulda S, Meyer E and Debatin KM: Metabolic inhibitors sensitize for CD95 (APO-1/Fas)-induced apoptosis by down-regulating Fas-associated death domain-like interleukin 1-converting enzyme inhibitory protein expression. Cancer Res 60: 3947-3956, 2000 .
31. Mathas S, Lietz A, Anagnostopoulos I, et al: c-FLIP mediates resistance of Hodgkin/Reed-Sternberg cells to death receptorinduced apoptosis. J Exp Med 199: 1041-1052, 2004.

32. Poulaki V, Mitsiades CS, Kotoula V, et al: Regulation of Apo2L/tumor necrosis factor-related apoptosis-inducing ligandinduced apoptosis in thyroid carcinoma cells. Am J Pathol 161: 643-654, 2002.

33. Longley DB, Wilson TR, McEwan M, et al: c-FLIP inhibits chemotherapy-induced colorectal cancer cell death. Oncogene 25: 838-848, 2006

34. Kathpalia VP, Mussak EN, Chow SS, et al: Genome-wide transcriptional profiling in human squamous cell carcinoma of the skin identifies unique tumor-associated signatures. J Dermatol 33: 309-318, 2006.

35. Riker AI, Enkermann SA, Fodstad O, et al: The gene expression profiles of primary and metastatic melanoma yields a transition point of tumor progression and metastasis. BMC Med Genomics 1: 13,2008 . 